\title{
Are dentists' phones a threat to patients?
}

\section{Dentists' perceptions of smart phone use in the clinical environment}

Br Dent J 2018; 225: 320-324 http://dx.doi.org/10.1038/sj.bdj.2018.642

Nowadays, smartphones seem to have become a basic necessity of life. With their growing popularity and use, we must ask how these devices may impact clinical dental settings. They are widely used for teaching, accessing resources and taking photos for diagnostic aids. However, do they really have a role in the dental surgery? Or are they merely a source of distraction and consequent error for clinicians?

In order to investigate this, Simon Wright and colleagues from the University of Salford conducted a study to determine the frequency of smartphone use by dentists and its perceived impact on patient safety. A questionnaire survey was constructed and sent to 216 dentists that had attended courses provided by an education provider in the North West of England from 2015-17. They were asked closed questions enquiring about their use of smartphones, alongside an open question regarding their views on what smartphone policies should include.

From the 107 responses received, it was found that $82.2 \%$ of the dentists had their phone in the surgery although $61.7 \%$ of them considered it as

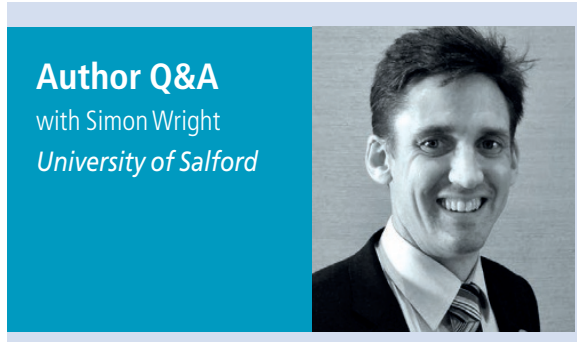

Why is it important to investigate smart phone use by dentists?

The dental practitioner is considered to be efficacious. However, dealing with the most intimate aspects of human life is not without error, and ultimately this may lead to consequences that impact on patient safety, for which the dental practitioner is held accountable.

Dentistry by its very nature involves complex manual tasks performed to a high degree of accuracy with a high risk for error. We now understand that most errors are attributable to human factors, including distractions and irritations in the clinical environment. With the rapid increase in the use of smart phones and their applications as a clinical aid, it is

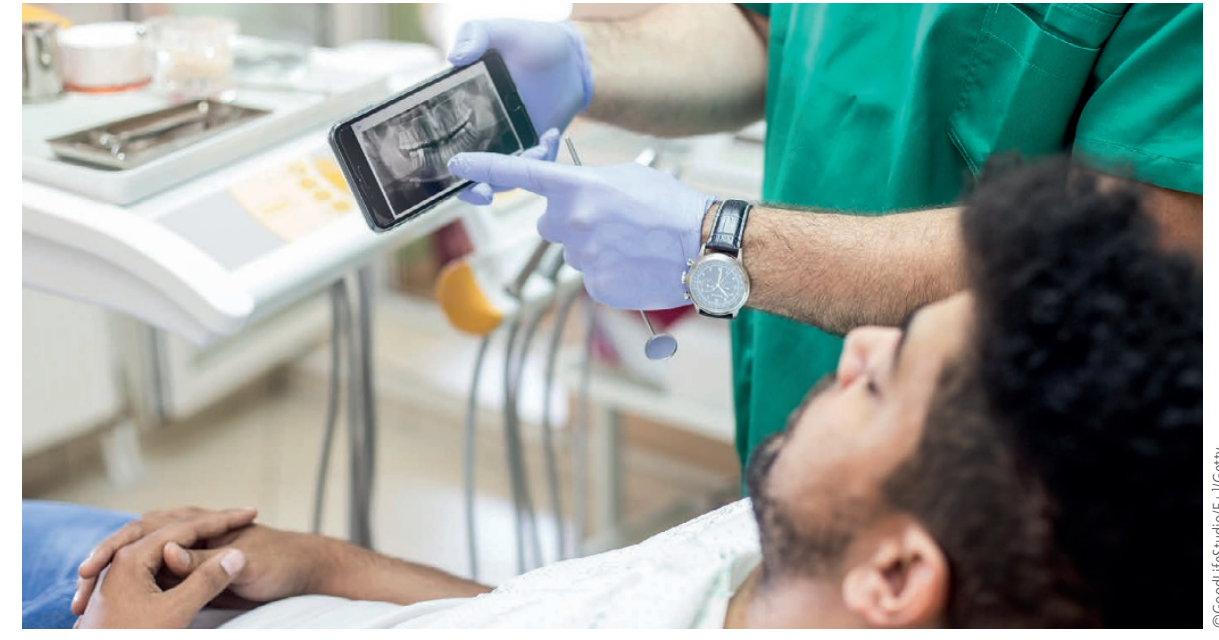

a distraction. Despite $88 \%$ of respondents keeping their phones on silent, the observation of frequent phone use was reported as $26.2 \%$ amongst dentists and $24.3 \%$ amongst nurses. On average, they were receiving three texts, four emails and one call per session. It was shown through average values that:

- $43.9 \%$ of dentists were unaware of being contacted

important to understand if they may contribute to the latent factors, that are the root causes of the errors that we make.

\section{Did anything surprise you?}

I was surprised that $88 \%$ of respondents had the smart phone present with them in the surgery, despite almost two thirds reporting that the smart phones distracted them from their clinical duties.

\section{What are the next steps?}

As we aim to make the practice of dentistry safer, it is vital to understand that the majority of the causes of error are related to human factors rather than technical ability or inadequate knowledge.

Currently we are concentrating our professional development on the use of technology and our intellectual capabilities. We need to identify, and learn about the latent causes of the errors that are made, and how to prevent them from occurring.
- $34.9 \%$ of respondents ignored the contactor

- $10.6 \%$ of them checked their phone but did not reply

- $6.8 \%$ choose to personally reply

- $3.8 \%$ asked another member of staff to reply.

With regards to the open question, there were mixed opinions such as:

- Twenty-eight dentists felt that they should be allowed to use their phone if on silent and out of view

- Twenty-four believed that smartphones should not be used under any circumstances

- Nineteen suggested that they can be used but not in front of patients

- Eight agreed that they should be used for accessing applications or the camera

- Others raised various issues such as data protection and cross infection, while some suggested specific scenarios under which their use should be permitted for example emergencies or allocated time slots.

Overall, the author concluded that using smartphones in dental settings may lead to distraction from clinical duties and their use should be in compliance to GDC standards, data protection and consent laws. As errors are indivisibly linked to human behaviour, it is important to regulate clinicians by establishing protocols and policies. This will ensure that the benefits can be achieved without compromise of patient care and safety.

By Sidra Ahmed Suleman 